\title{
Diversifikationsvorteile verbriefter Immobilienanlagen in einem Mixed-Asset-Portfolio
}

\author{
Roland Füss* \\ EBS Business School \\ Felix Schindler \\ ZEW Mannheim und Steinbeis Hochschule Berlin
}

\section{Einleitung}

Basierend auf Schätzungen der Volkswirtschaftlichen Gesamtrechnung für das Jahr 2008 beläuft sich das Vermögen des Immobilienbestandes in Deutschland auf rund 9.000 Mrd.€, wovon rund 2.706 Mrd. $€(30 \%)$ auf Gewerbe- und Infrastrukturbauten, rund 2.400 Mrd. $€(27 \%)$ auf bebauten Grund und Boden sowie rund $3.894 \mathrm{Mrd} . €(43 \%)$ auf Wohnbauten entfallen (Demary et al. 2009). In 2006 waren rund 5.033 Mrd.€ in privatem Haus- und Grundvermögen angelegt, was ca. 53\% des privaten Anlagevermögens in Deutschland darstellt. Anhand dieser Zahlen wird bereits deutlich, dass in Deutschland ein Großteil des Vermögens in direkte Immobilien allokiert ist. In den letzten Jahren haben auch indirekte Immobilienanlageformen in Form von geschlossenen oder offenen Immobilienfonds, Immobilienaktiengesellschaften oder von als steuerrechtlich ausgestalteten Real Estate Investment Trusts (REITs) sowie Real Estate Private Equity, Zertifikate und sonstige derivative Produkte stark an Bedeutung gewonnen. Daneben bieten Immobilien-Anlageformen mit Fremdkapitalcharakter wie z.B. Hypotheken-Pfandbriefe, verschiedene Typen von Mortgage-Backed Securities sowie Hypothekar- und Realkredite Anlegern eine weitere Möglichkeit, indirekt an Immobilien zu partizipieren. ${ }^{1}$

Die verstärkte Vermögensallokation in direkte Immobilien birgt erhebliche Risiken. So existiert am direkten Immobilienmarkt eine hohe Intransparenz wegen des individuellen Charakters von Objekten und deren fehlender Vergleichbarkeit sowie aufgrund des beschränkten Zugangs zu Transaktionsdaten. Des Weiteren erhöht die Vielzahl an regionalen und funktionalen Teilmärkten mit ihren spezifischen Angebots- und Nachfrageverhalten der Marktteilnehmer die Kosten der Informationsbeschaffung. Daneben entstehen Investoren aufgrund fehlender täglicher Liquidierbarkeit hohe Transaktionskosten, die wiederum eine geringe Umschlagshäufigkeit induzieren und zu einer relativ hohen Illiquidität des

\footnotetext{
*Korrespondenzadresse: Roland Füss, Union Investment Lehrstuhl Asset Management, EBS Business School, EBS Universität für Wirtschaft und Recht i. Gr., Gustav-Stresemann-Ring 3, 65189 Wiesbaden, Tel.: +49 (0)611 7102-1217, Fax: +49 (0)611 7102-101217, E-Mail: roland.fuess@ebs.edu und Felix Schindler, Zentrum für Europäische Wirtschaftsforschung (ZEW), Postfach 10 34 43, 68034 Mannheim, Tel: +49 (0) 621-1235-378, Fax: +49 (0) 621-1235-223, E-Mail: schindler@zew.de.

1. Siehe hierzu die Systematik in Tabelle 1 in Abschnitt 2.
} 


\section{Diversifikationsvorteile verbriefter Immobilienanlagen in einem Mixed-Asset-Portfolio}

Gesamtmarktes führen. ${ }^{2}$ Nach dem Kauf eines Objektes können gegenüber verbrieften Anlagen höhere steuerliche Belastungen wie auch beträchtliche Kosten der Verwaltung, insbesondere durch die starken Regulierungen des Mietmarktes für Wohnungen im Vergleich zu Anlagen in Finanzmarktprodukte, nachteilig auf die Rendite wirken. Darüber hinaus sorgen hohe Mindestanlagesummen dafür, dass direkte Immobilienanlagen fast ausschließlich für High Net Worth Individuals oder institutionelle Investoren in Frage kommen.

In Ergänzung oder als Alternative zu einem direkten Investment in Immobilien stehen Anlegern indirekte Immobilien-Anlageprodukte zur Verfügung, die es erlauben an der Entwicklung des Immobilienmarktes zu partizipieren ohne dabei, oder zumindest nur in abgeschwächter Form, den aus Anlegersicht nachteiligen Charakteristika einer direkten Immobilienbeteiligung ausgesetzt zu sein. Vorteile gegenüber direkten Immobilienanlagen ergeben sich für den Investor u.a. bei der Größen-, Fristen-, Risiko- und Informationstransformation, womit indirekte Immobilienbeteiligungen für an internationalen Kapitalmärkten agierende Investoren ein interessantes, alternatives Anlagevehikel darstellen. Dabei bietet sich dem Anleger eine in den letzten Jahren immer breiter werdende und sich auch in Deutschland zunehmend etablierende Produktpalette an, die es Investoren erlaubt, entsprechend ihrer Präferenzstruktur eine geeignete Anlageform zu wählen. Als global am stärksten wachsendes Immobilienanlageprodukt gelten dabei REITs, die ein weitgehend einheitlich geregeltes und steuertransparentes Anlagevehikel mit geringen Klumpenrisiken, hoher Liquidität, geringen Transaktionskosten und globalen Diversifikationsmöglichkeiten darstellen. Die bedeutendsten Partizipationsmöglichkeiten am Immobilienmarkt werden im nächsten Abschnitt kurz vorgestellt.

Da sich REITs nicht nur hinsichtlich ihrer Rendite/Risiko-Charakteristika, sondern insbesondere in Bezug auf Fungibilität und Transaktionskosten als vorteilhaft erweisen, ist das Ziel des Beitrages, das Diversifikationspotenzial dieser Anlageklasse in einem breit diversifizierten, internationalen Portfolio zu evaluieren. Hierbei wird vor allem auf den Langfristzusammenhang zwischen den Vermögensanlageklassen abgestellt, da sich, wie die Diskussion des dritten Abschnittes noch zeigen wird, für REITs gerade in der langen Frist ein Exposure zum Immobilienmarkt nachweisen lässt. Folglich wird vor dem Hintergrund des auf kurzfristigen Korrelationsstrukturen basierenden Mittelwert-Varianz-Ansatzes mit Hilfe der Analyse langfristiger Kointegrationsbeziehungen im vierten Abschnitt gezeigt, dass durch die Beimischung von REITs insbesondere für aktiv und langfristig orientierte Investoren positive Diversifikationseffekte in einem MixedAsset-Portfolio erzielt werden können. Der letzte Abschnitt fasst die wesentlichen Ergebnisse zusammen.

2. Die Folge dieser hohen Kosten sind in einzelnen Teilmärkten natürliche Marktzugangsbeschränkungen für einzelne Investoren, da die Informationskosten nicht durch eine entsprechende Rendite(-erwartung) kompensiert werden. Zusätzlich trägt die Illiquidität u.a. zur Trägheit des Marktes bei und lässt somit nur eine stark verzögerte Anpassung an neue Marktbedingungen zu. Aufgrund dieser institutionellen Gegebenheiten ist direkten Immobilienanlagen eine fehlende Markt- und Informationseffizienz im Sinne der Kapitalmarkttheorie zu konstatieren. Vgl. zur Markteffizienz direkter Immobilienmärkte u.a. Gau (1984), Case und Shiller (1989), Stein (1995), Genesova und Mayer (1997), Tirtiroglu (1992) sowie Schindler (2011) und die dort zitierte Literatur. 


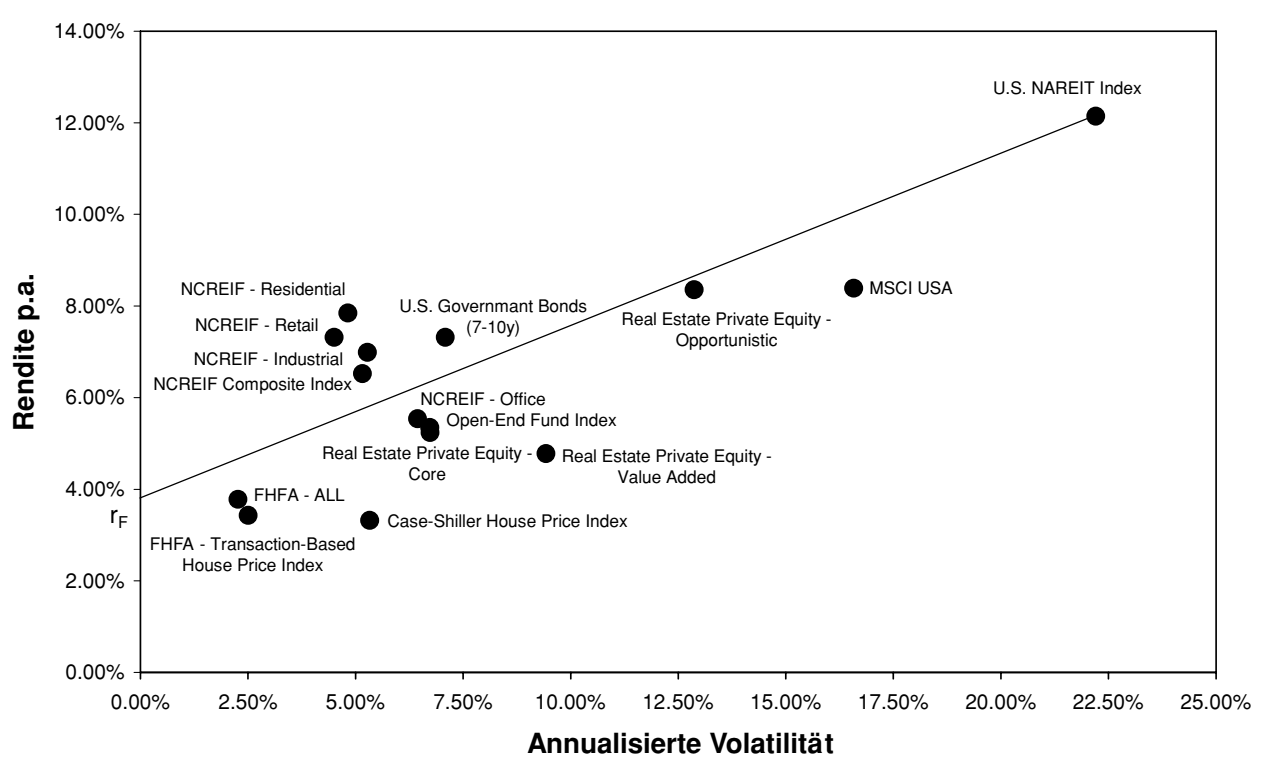

\begin{abstract}
Abbildung 1 Performancevergleich eigenkapitalbasierter Immobilienanlagen
Anmerkungen: Die Berechnungen basieren auf Quartalsrenditen für den Zeitraum von 1990/I bis 2009/IV (mit Ausnahme der Zeitreihe für den transaktionsbasierten FHFA Index mit Beginn 1991/II). Bei den verwendeten Indizes handelt es sich um Performance (Total Return) Indizes, bei denen neben

Preisentwicklungen auch Cash Flows in Form von Dividenden und Mietzahlungen berücksichtigt werden. Abweichend hiervon repräsentieren der FHFA Index und der Case Shiller House Price Index lediglich die Hauspreisentwicklung. Der risikolose Zins $r_{F}$ wurde anhand der dreimonatigen US-Treasury-Bill-Rate berechnet.
\end{abstract}

\title{
2. Investitionsmöglichkeiten in Immobilien
}

Neben der Kategorisierung von Immobilienanlageformen entsprechend ihrer Zuordnung zu Fremd- bzw. Eigenkapital erscheint gerade im Hinblick auf die Handelbarkeit und Liquidierbarkeit eine Einordnung gemäß der Eigenschaft „börsengehandelt” bzw. „nicht börsengehandelt” nahe liegend. Diese in der Literatur als vier Quadranten der Immobilienwirtschaft bezeichnete Systematisierung (Gordon 1994, Hudson-Wilson et al. 2005) in Abbildung 1 lässt die Attraktivität von Immobilienanlagen im Rahmen einer Kategorisierung in Eigen- und Fremdkapital sowie den damit verbundenen Diversifikationsgedanken erkennen. Die Bedeutung und Entwicklung der einzelnen Quadranten ist jedoch zwischen einzelnen Ländern höchst unterschiedlich ausgeprägt. ${ }^{3}$

Neben den oben erwähnten Nachteilen einer direkten Immobilienanlage kann in einem Vermögensportfolio deren hohe Mindestanlagesumme zudem negativ auf die realisierbaren Diversifikationseffekte wirken, indem tendenziell der optimale Portfolioanteil überschritten und folglich die Rendite-Risiko-Effizienz einer optimalen Anlage in Immobilien verfehlt wird. Hinzu kommt, dass - bedingt

3. Siehe ausführlich Hudson-Wilson et al. (2005) und Paramor und Wright (2006), die die Entwicklung der einzelnen Quadranten in den letzten Jahren und die Aufteilung des in Immobilienanlageformen investierten Kapitals in den USA und Australien untersuchen. 
Diversifikationsvorteile verbriefter Immobilienanlagen in einem Mixed-Asset-Portfolio

Tabelle 1 Die vier Quadranten der Immobilienwirtschaft ${ }^{4}$

\begin{tabular}{lll}
\hline & \multicolumn{1}{c}{ Eigenkapital } & \multicolumn{1}{c}{ Fremdkapital } \\
\hline nicht börsengehandelt & • direkte Immobilienanlage & • Hypothekarkredite \\
& $\bullet$ (offene Immobilienfonds) & $\bullet$ Realkredite \\
& $\bullet$ geschlossene Immobilienfonds & \\
• Real Estate Private Equity & \\
börsengehandelt & - Immobilienaktien & $\bullet$ Hypothekenpfandbriefe \\
& $\bullet$ REITs & $\bullet$ Mortgage-Backed Securities \\
\hline
\end{tabular}

durch die oben angesprochene Vielzahl an Teilmärkten - der Anleger bei einem Einzelinvestment hohen unsystematischen Risiken des Objektes ausgesetzt ist.

Der durch eine Beimischung einer Immobilie in ein Vermögensportfolio resultierende Risiko-Diversifikationseffekt wird vor allem durch die Eigenschaften des Realvermögensgutes hervorgerufen. Denn in ihrer Eigenschaft als Realvermögensgut bilden Immobilien gegenüber Geldvermögensgütern ein komplementäres Anlageprodukt und dienen zumindest aus theoretischer Sicht zur Diversifizierung, da sie im Vergleich zu Geldvermögen anderen Risiken unterliegen. Der Vorteil von sachwertgestützten Beteiligungen wird vor allem in ihrer Absicherung gegen Inflation gesehen (Wurtzebach et al. 1991, Mueller 2010). Diese attraktive Eigenschaft sollten aber - zumindest langfristig - auch indirekte Immobilienanlageformen besitzen, da diese ihre Erträge ebenfalls aus der Bestandshaltung von Immobilien (Mieterträge und Erträge aus Wertsteigerung) erzielen.

Zusammenfassend lässt sich festhalten, dass die Anlegerziele durch ein direktes Engagement in Immobilien nur unzureichend erfüllt werden können und sich die Frage stellt, ob andere Immobilien-Anlageprodukte existieren, die in der Lage sind, den Präferenzen und Zielen der Investoren besser nachzukommen bzw. Rechnung zu tragen. Allerdings zeigen in diesem Zusammenhang Pagliari et al. (2003, 2005), dass unter Berücksichtigung des Smoothing-Effektes (Geltner 1991) ${ }^{5}$ bei direkten Immobilien und des Einflusses von Leverage bei REITs sowie durch eine Bereinigung der Sektorenkonzentration in beiden Anlagekategorien eine deutliche Annäherung im Rendite-Risiko-Spektrum für den US-amerikanischen Markt im Zeitraum von 1981 bis $2001 \mathrm{zu}$ erkennen ist. Auch Riddiough et al. (2005) finden für die USA im Betrachtungszeitraum von 1980 bis 1998 eine wachsende Konvergenz zwischen direktem und indirektem Immobilienmarkt. Als mögliche Abweichungen in der Anlageperformance führen die Autoren lediglich fehlende Anpassungen in den Risikofaktoren Geographie und Liquidität an.

4. Die Einordnung offener Immobilienfonds gilt aufgrund ihres zusätzlichen Handels an Wertpapierbörsen nur bedingt. Es ist jedoch $\mathrm{zu}$ beobachten, dass die an den Finanzmärkten festgestellten Kurse für offene Immobilienfonds sich in der Spanne zwischen Ankaufs- und Verkaufskurs der KAG des jeweiligen Fonds bewegen. Ein festgestellter Kurs außerhalb dieser Spanne würde zu Arbitrage-Möglichkeiten führen, da die KAG verpflichtet ist, Anteile zu ihrem Net Asset Value (NAV) zurückzunehmen bzw. zu ihrem NAV zzgl. Ausgabeaufschlag auszugeben.

5. Der Smoothing-Effekt resultiert aus einer fehlenden transaktionsbasierten Bewertung von direkten Immobilien. Durch die angewandte Bewertungsmethodik, die im Wesentlichen auf diskontierten Zahlungsströmen basiert, ergibt sich eine Persistenz in der Wertermittlung. Im statistischen Sinne wird hierdurch eine (positive) Autokorrelation in den Renditen erzeugt und folglich das Risiko unterschätzt bzw. verzerrt wiedergegeben. 
Das Ziel offener Immobilienfonds ist es, die Nachteile eines direkten Immobilieninvestments für den einzelnen Anleger $\mathrm{zu}$ überwinden und „dem Anteilsscheininhaber langfristig eine angemessene laufende Verzinsung aus regelmäßig erwirtschafteten Erträgen aus Immobilien und liquiden Mitteln (Wertpapieren und Bankguthaben) sowie eine möglichst kontinuierliche Wertentwicklung bei gleichzeitiger Optimierung des steuerlichen Ergebnisses zu bieten" (Alda und Lassen 1998, S. 93).

Durch den Erwerb von Anteilsscheinen eines offenen Immobilienfonds ist der Anleger schon mit relativ geringen Mindestanlagevolumina an den Erträgen und der Wertentwicklung aus den im Sondervermögen des Fonds enthaltenen Immobilien und sonstigen Vermögensgegenständen beteiligt. Im Gegensatz zum geschlossenen Immobilienfonds ist weder die Anzahl der Anleger noch das Fondsvolumen begrenzt und die Kapitalanlagegesellschaft (KAG) ist grundsätzlich dazu verpflichtet, börsentäglich einen Wert der Anteilsscheine festzustellen sowie diese auszugeben und zurückzunehmen. Während sich dies lange Zeit als unproblematisch erwies, musste Ende 2005 erstmals in Deutschland eine KAG die Rücknahme von Anteilsscheinen aussetzen. Im Zuge der Finanzmarktkrise ab 2008 gerieten weitere offene Immobilienfonds in Liquiditätsschwierigkeiten und waren durch die hohen Mittelabflüsse gezwungen, die Rücknahme von Anteilsscheinen auszusetzen. Dies führte in der Folge zu einer heftigen Diskussion um die rechtliche Ausgestaltung des Anlageproduktes und offenbarte dem Anleger die mit einer solchen Investition verbundenen Risken.

Eine Besonderheit von offenen Immobilienfonds gegenüber anderen Investmentfonds liegt insbesondere in der Wertermittlung der Fondsanteile. Diese erfolgt nicht über die Börsen, sondern wird von Sachverständigen für Immobilienbewertung durchgeführt, die in regelmäßigen Abständen eine Bewertung der sich im Fondsbesitz befindlichen Immobilien durchführen. ${ }^{6}$

Einige offene Immobilienfonds werden inzwischen auch an deutschen Börsenplätzen gehandelt. Dies ermöglicht dem Anleger, jederzeit seine Anteile zu veräußern bzw. neue zu erwerben, wobei die Anteilsscheine bei Fonds, deren Anteilsscheinrücknahme durch die KAG ausgesetzt ist, unter Umständen mit deutlichen Abschlägen auf den von der KAG ausgewiesenen Wert notieren. Bei Fonds, deren Rücknahme und Ausgabe nicht ausgesetzt sind, orientiert sich der Börsenkurs allerdings stark am von der KAG festgestellten Wert, da sonst durch die Verpflichtung der KAG zur Rücknahme bzw. Ausgabe von Anteilsscheinen Arbitrage-Möglichkeiten entstünden.

Insgesamt besitzen die offenen Immobilienfonds mit einem Vermögen von rund 87,1 Mrd. € Ende 2009 einen Anteil am gesamten Vermögen deutscher Publikumsfonds in Höhe von rund 13,4\% (BVI 2010, S. 4). Mit Ausnahme der

6. Statistisch zeigt sich das vergleichsweise geringe Anlagerisiko durch die niedrige Volatilität der Fonds-Renditen. Allerdings gilt es hierbei zu beachten, dass der Ausweis der geringen Volatilitäten durch die ebenfalls gesetzlich vorgegebene Bewertungsmethodik und Messtechnik bedingt ist und nicht das fundamentale Risiko der Wertschwankungen am Immobilienmarkt zum Ausdruck bringt. Dieser sog. Smoothing-Effekt (Geltner 1991) führt somit zu einem nicht adäquaten Ausweis des tatsächlich mit der Anlage in einen offenen Immobilienfonds verbundenen Risikos. Gegenüber geschlossenen Immobilienfonds erweisen sich offene Immobilienfonds durch die Veröffentlichung der Bewertungsergebnisse und Eigenschaften der Einzelimmobilien sowie der Einzelaufstellung der unterschiedlichen Renditekomponenten als transparenter und sind für den Anleger mit einem geringeren Verwaltungsaufwand verbunden. 


\section{Diversifikationsvorteile verbriefter Immobilienanlagen in einem Mixed-Asset-Portfolio}

Jahre 1990, 2000, 2005 und 2006 weisen offene Immobilienfonds positive jährliche Mittelzuflüsse aus und das in offene Immobilienfonds investierte Vermögen hat sich seit 1990 mehr als verzehnfacht.

Im Vergleich $\mathrm{zu}$ offenen Immobilienfonds zeichnen sich geschlossene Immobilienfonds vor allem dadurch aus, dass das Volumen der einzelnen Fonds begrenzt und die Rückgabe der Anteile durch den Anleger nicht jeder Zeit möglich ist. Zwar wird dem Anleger ein Sonderkündigungsrecht eingeräumt und es existieren vereinzelt Sekundärmärkte für die Fondsanteile, allerdings ist das Volumen an diesen Märkten sehr gering, die Preisabschläge vergleichsweise hoch und die Märkte sind nur sehr eingeschränkt funktionsfähig. Ein liquider Zweitmarkt für die Fondsanteile über eine Börse existiert also nicht. Lange Zeit lag die Attraktivität dieses Anlageproduktes vor allem in seiner steuerlichen Begünstigung. Diese wurde in den letzten Jahren allerdings zunehmend zurückgefahren, wodurch sich in der Zukunft die Fokussierung auf die ökonomischen Eigenschaften der FondsImmobilien durch das Fondsmanagement erhöhen dürfte.

Neben der Attraktivität ausländischer Immobilienmärkte zogen geschlossene Auslands-Immobilienfonds vor allem durch ihre steuerlichen Vorteile das Interesse deutscher Anleger auf sich. Während Mitte der 1990er Jahre der Auslandsanteil noch eine untergeordnete Rolle spielte, stieg das auf ausländische Objekte entfallende Platzierungsvolumen bis zum Jahr 2005 auf über 60\% an. Allerdings haben solche Auslands-Investments auch immer wieder das mit dieser Anlageform einhergehende Risiko deutlich gemacht.

Das Anlagevehikel Real Estate Private Equity (REPE) stellt eine branchenspezifische Variante des allgemeinen Investmentsegmentes Private Equity dar und besitzt daher auch weitgehend die gleichen Eigenschaften. REPE-Beteiligungskapital unterliegt einer geringen gesetzlichen Regulierung. Charakteristisch für REPEInvestments sind deren hoher Fremdkapitalanteil, die gegenüber der Immobilie kurze Laufzeit von REPE-Fonds, die vergleichsweise hohen Mindestanlagevolumina, die Fokussierung auf Objekte mit einem hohen Wertsteigerungspotential bei gleichzeitig hohem Risiko sowie die allgemein hohe Risikobereitschaft von REPEInvestoren. Die Börsennotierung wird lediglich als Exit-Option wahrgenommen. Durch diese Eigenschaften eignet sich das Anlageprodukt vor allem für wohlhabende Privatpersonen und institutionelle Anleger.

Im Gegensatz zu dem steuertransparenten und meist ebenfalls börsennotierten Anlageprodukt des REITs ist die (börsengehandelte) Immobilienaktiengesellschaft nicht gesetzlich definiert und besitzt daher gegenüber anderen Aktiengesellschaften in ihrer Ausgestaltung keine rechtlichen oder steuerlichen Besonderheiten. Die unterschiedliche Auslegung des Begriffs „Immobilien-AG” führt jedoch dazu, dass unter dieser Kategorie eine Vielzahl von in ihrem Kerngeschäft und Tätigkeitsgebiet höchst verschiedenen Unternehmen, wie ImmobilienBestandshalter, Projektentwickler und Dienstleister, zusammengefasst wird. ${ }^{7}$

Während aus Anlegersicht Investitionen in börsengehandelte Immobilien-AGs sich in ihren Eigenschaften (geringe Transaktionskosten, Publizitätspflichten, tägliche Bewertung durch den Markt etc.) im Allgemeinen nicht von Anteilsscheinen aus anderen Aktiensegmenten unterscheiden, wirkt der oft geringe Free Float einschränkend auf die Fungibilität bei Immobilien-AGs.

7. Vgl. zu dieser begrifflichen Abgrenzung Rehkugler (2000, S. 231) sowie Ellwanger und Geiger (2000, S. 52ff.). 
Bzgl. der mit der Anlage verbundenen Risiken - in Form der Volatilität der Renditen - ist festzustellen, dass diese sich für den Investor - zumindest gegenüber einer Anlage in offene Immobilienfonds - als wesentlich größer erweisen. Neben dem Risiko, das aus der Investition in den Immobilienmarkt resultiert und dem das Unternehmen durch seine Geschäftstätigkeit unmittelbar unterworfen ist, besteht als zweite Risikoquelle das Kapitalmarktrisiko. Die geringen Mindestanlagesummen ermöglichen es jedoch auch Privatanlegern durch ein breit aufgestelltes und diversifiziertes Portfolio die unsystematischen Risiken weitgehend $\mathrm{zu}$ meiden und sich lediglich den systematischen Risiken auszusetzen.

Eine spezielle Form der Immobilienaktiengesellschaft stellen Real Estate Investment Trusts dar. REITs grenzen sich insbesondere durch ihre steuerliche Behandlung von der allgemeinen Form einer Immobilienaktiengesellschaft ab. Die Anforderungen an einen REIT unterliegen starken Restriktionen durch den Gesetzgeber, der ähnlich zum offenen Immobilienfonds in Deutschland dem Anleger ein relativ sicheres und transparentes Anlagevehikel mit regelmäßigen Ausschüttungen zur Verfügung stellen will. Die steuerliche Grundidee bei REITs besteht in der Besteuerung der Ausschüttungen auf der Anlegerebene und nicht auf der Ebene des Trusts. Im Hinblick auf die zentralen Anlegerziele - Rendite, Sicherheit, Liquidierbarkeit und Verwaltbarkeit (Schmidt-von Rhein 1996, S. 186f.) - ist in der steuerlichen Transparenz der wesentliche Unterschied im Vergleich zu den Immobilien-AGs zu sehen.

Um nach US-amerikanischem Steuerrecht den „tax-exempt-Status” eines REITs erreichen zu können, sind durch die Gesellschaft verschiedene Bedingungen zu erfüllen, die in der Literatur fast einheitlich nach Vermögens-, Einkommens-, Ausschüttungs- und Organisationsanforderungen kategorisiert werden. ${ }^{8}$ Die Erfüllung der Bedingungen zur Qualifizierung für den REIT-Status kann von Unternehmen mit verschiedenen Geschäftstätigkeiten erbracht werden. Daher wird zwischen sog. Equity-, Mortgage- und Hybrid-REITs differenziert. ${ }^{9}$ Die vorrangige und am weitesten verbreitete Art von REITs bilden die Equity-REITs, die in den USA Ende 2010 mit einer Marktkapitalisierung von rund 358,9 Mrd. US-Dollar einen Marktanteil von über $90 \%$ besitzen und somit die dominante REIT-Anlageform darstellen. Ihre Geschäftsgrundlage stellt das Poolen von Kapital zum Kauf von Immobilien zur langfristigen Erzielung von Mieteinnahmen (Bestandshaltung) dar.

Inzwischen bestehen weltweit in 35 Ländern REIT-Gesetzgebungen bzw. rechtliche Rahmenbedingungen für REIT-ähnliche Konstruktionen (Vgl. EPRA 2009). Die rechtliche und steuerliche Ausgestaltung in den einzelnen nationalen REIT-Strukturen und -Gesetzgebungen orientiert sich weitestgehend an

8. Vgl. hierzu ausführlich Brueggeman und Fisher (2008, S. 622), EPRA (2009, S. 316ff.), Fass et al. (2005, S. 768), Chan et al. (2003, S. 37) sowie Downs und Hartzell (1995, S. 630f.). In EPRA (2009) findet sich des Weiteren eine detaillierte Beschreibung nationaler Ausgestaltungen von REIT-Gesetzgebungen und eine vergleichende Gegenüberstellung von 35 Ländern mit REIT-Strukturen weltweit.

9. Als Equity-REIT qualifiziert gelten REITs, die mindestens $75 \%$ ihres Vermögens in Immobilienbesitz investiert haben. Mortgage-REITs müssen mindestens 75\% ihrer Assets in durch Immobilien besicherte Grundpfandrechte anlegen. Vgl. Fass et al. (2005), S. 157 und Chan et al. (2003), S. 17. Sind weder die eine noch die andere Quote erreicht, erfolgt eine Einordnung als Hybrid-REIT. Vgl. Lee und Chiang (2004, S. 111) und Fass et al. (2005, S. 167). 


\section{Diversifikationsvorteile verbriefter Immobilienanlagen in einem Mixed-Asset-Portfolio}

den Regelungen in den USA. ${ }^{10}$ Somit existiert international ein transparentes und in seinen Rahmenbedingungen fast einheitliches Immobilienanlageprodukt, das sowohl bei privaten als auch bei institutionellen Investoren auf eine wachsende Resonanz und ein verstärktes Interesse stößt. ${ }^{11}$ Die zu beobachtende Verschiebung in den Anteilen der weltweiten Marktkapitalisierung zu Gunsten der asiatischen und europäischen Märkte und zu Lasten des nordamerikanischen Marktes in den letzten Jahren bestätigt die These einer zunehmenden globalen Ausdehnung dieses Marktsegmentes. Diese Entwicklung spiegelt auch die weltweite Suche nach gegenüber Aktien und Anleihen alternativen Anlagemöglichkeiten und die damit gerade in den letzten Jahren gestiegene Attraktivität der Immobilienaktienmärkte für Investoren wider.

Zusammenfassend werden in Abbildung 1 die unterschiedlichen eigenkapitalbasierten Immobilienanlageformen gemäß ihres Rendite-Volatilitäts-Verhältnisses gegenübergestellt. Unter Verwendung von Quartalsrenditen zeigt sich für den US-amerikanischen Markt, dass im Betrachtungszeitraum von 1990/I bis 2009/IV lediglich die direkten Gewerbe-, mit Ausnahme von Büroimmobilien, und gewerblichen Wohnimmobilien (NCREIF) sowie die Staatsanleihen mit 710jähriger Laufzeit den nationalen REIT-Markt outperformen. Sowohl die offenen Immobilienfonds als auch Real Estate Private Equity (Core, Opportunistic und Added Value) sowie die Indizes für Wohnimmobilienpreise der Federal Housing Finance Agency (FHFA) und nach Case-Shiller liegen im $\mu$ - $\sigma$-Raum unterhalb der Verbindungslinie zwischen risikolosem Zins $\left(\mathrm{r}_{\mathrm{F}}\right)$ und dem NAREIT REIT-Index. Für die beiden Indizes mit Bezug zum Wohnungsmarkt ist anzumerken, dass lediglich die Wertentwicklung und keine Mietzahlungen berücksichtigt werden. Unterstellt man eine Mietrendite von lediglich 2-3\%, so würde sich der Anlageerfolg dem der NCREIF-Indizes annähern. Des Weiteren deckt der Case-Shiller Composite Index nur zehn Städte (Boston, Chicago, Denver, Las Vegas, Los Angeles, Miami, New York, San Diego, San Francisco und Washington, D.C.) ab. Schließlich weist auch der nationale Aktienmarkt (MSCI USA) gegenüber dem REIT-Markt ein nachteiliges Rendite-Risiko-Verhältnis auf.

Neben den beschriebenen Immobilienanlageprodukten mit Eigenkapitalcharakter existieren sowohl börsennotierte als auch nicht-börsennotierte Immobilienanlageformen mit Fremdkapitalcharakter. Allerdings handelt es sich im Wesentlichen um Produkte, die in ihrer Grundstruktur auch außerhalb des Immobiliensektors bekannt sind. Daher wird die Darstellung der entsprechenden Produkte an dieser Stelle kurz gehalten.

Im Unterschied zu den Anlageformen mit Eigenkapital ist den Anlageformen mit Fremdkapital gemein, dass sich ihre Rendite nicht unbedingt direkt am Erfolg des Projektes bzw. den durch die Immobilie generierten Einnahmen orientiert und das mit der Anlage verbundene Risiko in der Regel geringer ausfällt. Zum einen ist das zur Disposition stehende Kapital auf die Einlage begrenzt und zum

10. Einen Überblick über die Ausgestaltung der REIT- und REIT-ähnlichen Konzepte im internationalen Vergleich liefern EPRA (2009), Fass et al. (2005, S. 768ff.) sowie Eichholtz und Kok (2007, S. 65f.) für den europäischen Markt.

11. Die weltweite Marktkapitalisierung von durch die EPRA-Indizes erfassten Immobilienaktiengesellschaften - ausgehend von einem Wert von ca. 331 Mrd. $€$ im Jahre 2001 - hat sich innerhalb von fünf Jahren auf einen Wert von ca. 687 Mrd. $€$ gegen Ende des Jahres 2006 mehr als verdoppelt. Im Oktober 2010 betrug die Marktkapitalisierung ca. 553 Mrd. $€$. 
anderen werden die Ansprüche des Fremdkapitals im Falle der Zahlungsunfähigkeit des Schuldners gegenüber den Eigenkapitaleinlagen vorrangig behandelt und entgolten. Gleichzeitig sind aber auch die für den Investor mit der Anlage verbundenen Mitspracherechte sehr begrenzt.

Das originäre Produkt ist hierbei ein Immobilienkredit im Allgemeinen bzw. ein Hypothekarkredit im Speziellen. Allerdings besitzen beide Kreditarten analog zu einer Investition in eine direkte Immobilie ungewünschte Eigenschaften für den Anleger bzw. entsprechen seinen Zielen in der Regel nur unzureichend. Alternativen bieten daher deutsche Hypotheken-Pfandbriefe sowie Mortgage-Backed Securities (MBS). Beiden Produkten liegen originär in der Regel mehrere (Hypothekar-) Kredite zu Grunde. Ebenso ist beiden Produkttypen gemein, dass sie eine Spezialform eines am Kapitalmarkt allgemeiner gefassten Vehikels darstellen. Während MBS eine Spezialform von Asset-Backed Securities darstellen, handelt es sich bei den Hypotheken-Pfandbriefen um eine besondere Form des deutschen Pfandbriefs, der wiederum die deutsche Spezialform des jeweils national individuell ausgeprägten Covered Bonds darstellt. Im internationalen Vergleich und gegenüber MBS wird der deutsche Hypotheken-Pfandbrief als relativ sicheres Anlageinstrument eingestuft, bei dem es auch im Zuge der Finanzmarktkrise zu keinen Ausfällen kam. Dies ist vor allem auf seine rechtliche Ausgestaltung und den dahinter stehenden Sicherheitsgedanken zurück zu führen.

Unter Vernachlässigung der fremdkapitalbasierten Anlagevehikel hat sich gezeigt, dass insbesondere verbriefte Immobilienanlagen in Form von REITs hinsichtlich ihrer Ausgestaltung und in Bezug auf die anfallenden Informationsund Transaktionskosten gegenüber anderen Anlageformen als vorteilhaft einzuschätzen sind. Vor dem Hintergrund der bereits geführten Diskussion zur Rendite-Risiko-Einordnung direkter und verbriefter Immobilienanlagen, stellt sich jedoch für einen Anleger, der einerseits an der Wertentwicklung des Assets Immobilie partizipieren und andererseits die Vorteile von REITs gegenüber einer Direktinvestition in eine Immobilie realisieren möchte, die Frage, ob er bei einer Investition in REITs eher ein Exposure zum Immobilienmarkt erhält oder es sich um eine Investition in gewöhnliche Aktien handelt. In diesem Zusammenhang stellt sich für den Anleger als zweite Frage, ob direkte und indirekte Immobilienanlagen einen zeitlichen Gleichlauf aufweisen und ob bzw. welche Prognosekraft der eine Markt für den anderen besitzt.

\section{REITs - Immobilie oder Aktie?}

Aus theoretischer Sicht haben direkte Immobilienanlagen und REITs gemein, dass die zu Grunde liegenden Vermögensgegenstände, in die der Anleger investiert, in beiden Fällen Immobilien sind und somit langfristig beide Anlagen eine ähnliche Wertentwicklung aufweisen sollten. Allerdings handelt es sich bei REITs (mehrheitlich) um börsengelistete Unternehmen, die einer täglichen Bewertung an den Aktienmärkten unterliegen und sich somit auch den Marktschwankungen an den Aktienmärkten nicht vollkommen entziehen können. Dies führt zu der Hypothese, dass REITs in der kurzen Frist einen deutlich stärkeren Gleichlauf mit Aktien aufweisen als mit Immobilien, dieser Zusammenhang sich aber in der langen Frist abschwächt und von einem zunehmenden Gleichlauf zwischen REITs und Immobilien dominiert wird. Bzgl. 


\section{Diversifikationsvorteile verbriefter Immobilienanlagen in einem Mixed-Asset-Portfolio}

der zweiten aufgeworfenen Fragestellung ist $\mathrm{zu}$ erwarten, dass eine Prognosekraft für den entsprechenden Immobilienmarkt vom REIT-Markt ausgehen sollte. Dies ist damit zu begründen, dass Immobilienmärkte zum einen durch eine hohe Ineffizienz und stark zyklisches Verhalten gekennzeichnet sind (Phyrr et al. 1999), während börsengehandelte Kapitalmarktprodukte als weitgehend effizient bezeichnet werden können, da neue Informationen deutlich schneller in den Preisen verarbeitet werden sowie die Umschlagshäufigkeit und die Transparenz höher sind. Im Gegensatz zu Marktbewertungen bei REITs basieren durch Indizes abgebildete Preisentwicklungen an den Immobilienmärkten häufig nicht auf Marktbewertungen, die aus Transaktionen resultieren, sondern auf Preiseinschätzungen durch Immobilienbewerter (Geltner 1993). Dies führt zum einen dazu, dass sich die Bewertungen erst mit einer gewissen zeitlichen Verzögerung in den Indizes widerspiegeln und zum anderen die Bewertungen hohe Persistenzen aufweisen, was sich im Smoothing-Effekt niederschlägt und aus statistischer Sicht zu hohen Autokorrelationen führt (Quan und Quigley 1989).

Aus den theoretischen Überlegungen lässt sich daher die Schlussfolgerung ableiten, dass bei der Diskussion, ob REITs eher das Verhalten von Aktien oder von direkten Immobilien zeigen, der zeitlichen Dimension eine bedeutende Rolle zukommt. Kurzfristig ist anzunehmen, dass REITs durch ihre Börsennotierung in ihrem Verhalten eine große Ähnlichkeit zu Aktien besitzen, während in der langen Frist davon auszugehen ist, dass sich dieser Zusammenhang abschwächt und sich ein stärkerer Gleichlauf zwischen REITs und direkten Immobilienanlagen zeigt. Ebenfalls ist zu erwarten, dass indirekte Immobilienanlagen Vorlaufeigenschaften gegenüber direkten Immobilienanlagen aufweisen.

Neben der rein theoretischen Diskussion überprüfen Morawski et al. (2008) und Oikarinen et al. (2009) die aufgeworfenen Fragestellungen für den USamerikanischen Markt auch empirisch. Als Datengrundlage dient beiden Analysen der NCREIF-Index für den direkten Immobilienmarkt bzw. der NAREIT-Index für den indirekten Immobilienmarkt (REIT-Markt). Der US-amerikanische Aktienmarkt wird in beiden Analysen durch einen breiten Aktienmarktindex abgebildet (S\&P 500 bzw. Datastream US-Aktienmarktindex). Beide Analysen beziehen sich auf einen ähnlichen Zeitraum, der sich von 1978 bis 2006 bzw. 2008 erstreckt. Zur Analyse der kurzfristigen Zusammenhänge zwischen den drei Anlageklassen wird in beiden Untersuchungen auf die Korrelationsanalyse zurückgegriffen, während die Analyse der langfristigen Abhängigkeiten auf Basis der Kointegrationsanalyse nach Johansen $(1988,1991)$ erfolgt. Durch den ähnlichen Studienaufbau, einen ähnlichen Untersuchungszeitraum und die Verwendung ähnlicher Indexreihen kommen die beiden Untersuchungen zu qualitativ gleichen Ergebnissen.

Es zeigt sich, dass die Korrelation zwischen dem NAREIT-Index und dem US-amerikanischen Aktienindex auf Basis von Quartalsdaten deutlich höher ist als zwischen dem NAREIT-Index und dem NCREIF-Index. Werden allerdings Zeiträume von länger als einem Jahr betrachtet, ist zu erkennen, dass die Korrelation zwischen dem NAREIT-Index und dem NCREIF-Index deutlich ansteigt, während sich der Zusammenhang zwischen dem NAREIT-Index und dem USamerikanischen Aktienindex stark abschwächt bzw. der Korrelationskoeffizient sogar leicht negativ wird. Bei der Analyse der Vorlaufeigenschaften von indirekten gegenüber direkten Immobilienanlagen mittels Korrelationen wird deutlich, dass die Korrelation zwischen beiden Anlageklassen auf Basis der 3- und 5-Jahresrenditen 


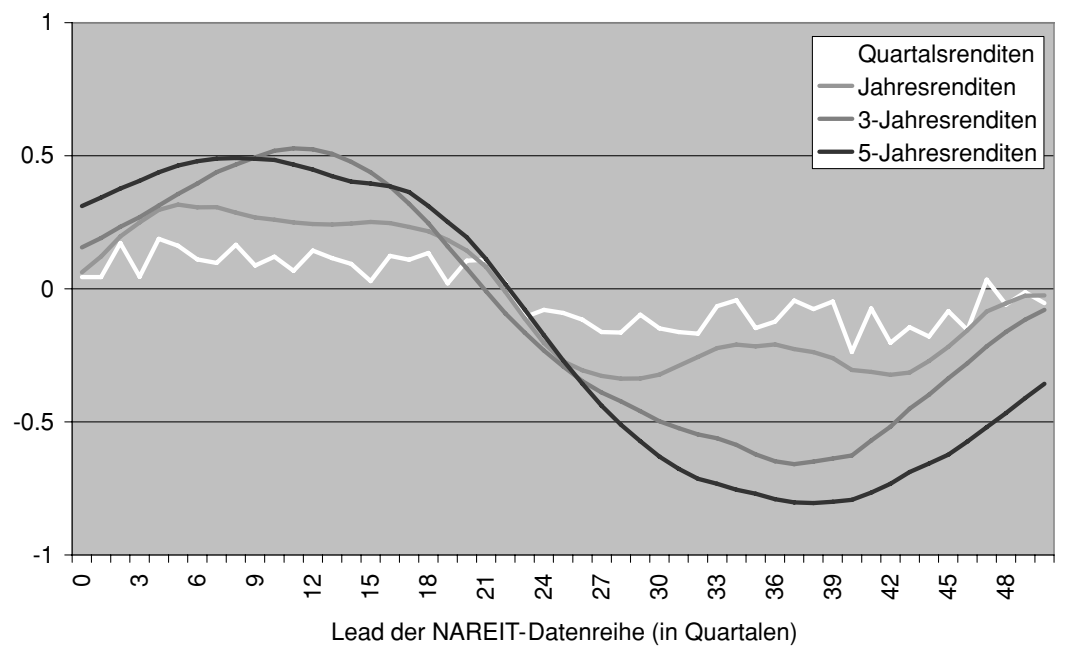

Abbildung 2 Renditekorrelationen zwischen NCREIF- and NAREIT-Index in Abhängigkeit vom Lead in den NAREIT-Daten

Quelle: Morawski et al. (2008, S. 111).

Anmerkungen: Die Abbildung zeigt die Korrelationskoeffizienten zwischen NAREIT- und

NCREIF-Index, d.h. zwischen dem verbrieften und direkten US-Immobilienmarkt, unter Berücksichtigung der Lead-Struktur für den NAREIT-Index (auf der $x$-Achse). Es zeigt sich, dass die Ergebnisse mit Ausnahme der Quartalsrenditen für alle Halteperioden ähnlich sind: Zunächst steigt die Korrelation bis zu einem Maximum an, um dann zu sinken. Die Länge des optimalen Leads hängt allerdings vom Anlagehorizont ab. Für jährliche, 3-Jahres- und 5-Jahres-Renditen wird die maximale Korrelation bei einem Vorlauf von 5, 11 und 8 Quartalen erreicht.

bei einer Verzögerung von bis zu 11 bzw. 8 Quartalen deutlich ansteigt (siehe Abbildung 2). ${ }^{12}$

Die Ergebnisse der Kointegrationsanalyse bestätigen qualitativ die Befunde aus der Korrelationsanalyse. So finden Morawski et al. (2008) sowohl für den gesamten Beobachtungszeitraum als auch für zwei Subperioden, die sich aus einem Strukturbruch Anfang der 1990er Jahre ergeben, ${ }^{13}$ jeweils eine Kointegrationsbeziehung zwischen den drei analysierten Zeitreihen. Interessant ist hierbei, dass sich der Zusammenhang zwischen dem REIT-Markt und dem direkten Immobilienmarkt als konstant erweist, während der Zusammenhang zwischen dem Aktien- und REIT-Markt in der zweiten Subperiode zusammenbricht. Aus dem Fehlerkorrekturmodell und ergänzenden Exogenitätstests bestätigt sich auch die durch die Korrelationsanalyse gewonnene Erkenntnis, dass der

12. Da es sich beim NCREIF-Index um einen bewertungsbasierten Index handelt, der neben weiteren Nachteilen auch erst verzögert die Preisentwicklung am entsprechenden Immobilienmarkt abbildet, führen Morawski et al. (2008) die Analysen zusätzlich auch auf Basis des entglätteten, transaktionsbasierten Index nach Fisher und Geltner (2000) durch. Die Ergebnisse erweisen sich allerdings als weitgehend robust, auch wenn sich der Vorlauf des NAREIT-Index leicht abschwächt.

13. Der diagnostizierte Strukturbruch fällt in einen Zeitraum, der in der Literatur häufig auch als Beginn der „New REIT Era“ gesehen wird. Im Vergleich zu Morawski et al. (2008) finden Oikarinen et al. (2009) zwar keinen Strukturbruch, stellen aber ebenfalls fest, dass zumindest temporär eine starke Abweichung des langfristigen Zusammenhangs zwischen direkten und indirekten Immobilienanlagen besteht. 


\section{Diversifikationsvorteile verbriefter Immobilienanlagen in einem Mixed-Asset-Portfolio}

direkte Immobilienmarkt dem REIT-Markt nachläuft und einen signifikanten Beitrag zum Anpassungsprozess an die langfristige Gleichgewichtsbeziehung leistet. Somit stehen die empirischen Erkenntnisse weitgehend in Einklang mit den theoretischen Überlegungen zum Zusammenhang zwischen direkten und indirekten Immobilienanlagen. Als Begründung für die im Zeitablauf abnehmenden Korrelationen zwischen dem Aktien- und REIT-Markt für kurzfristige Halteperioden kann die zunehmende Bedeutung von Immobilienaktien als separate Asset-Klasse angeführt werden. Die in der langen Frist zu beobachtenden höheren Korrelationen zwischen direkten und verbrieften Immobilien verdeutlichen hingegen die Qualifizierung von Immobilien als langfristiges Investment.

Als Fazit ist festzuhalten, dass der Anlagehorizont die entscheidende Determinante bei der Diskussion der oben aufgeworfenen Fragestellung darstellt: Während sich REITs einerseits den Einflüssen der Aktienmärkte kurzfristig nicht einziehen können, verfestigt sich andererseits mit zunehmendem Anlagehorizont ein starker Zusammenhang in der Entwicklung von REITs und direkten Immobilienanlagen. Dieses Ergebnis steht in Einklang mit den Analysen von Geltner et al. (1995), Eichholtz und Hartzell (1996) und Pagliari et al. (2005), die zeigen, dass die Unterschiede in den Rendite- und Risikoeigenschaften von REITs und direkten Immobilienanlagen deutlich geringer ausfallen, wenn die (Ent-) Glättungsproblematik bei bewertungsbasierten Indizes, der Verschuldungsgrad bei indirekten Anlagen und der differierende Immobilien-Mix von NCREIF- und NAREIT-Index in der Analyse Berücksichtigung finden. Ebenfalls deuten die Befunde von Morawski et al. (2008) und Oikarinen et al. (2009) darauf hin, dass die Entwicklung von REITs derjenigen von direkten Immobilienanlagen voraus läuft, was u.a. auf Marktineffizienzen und die angesprochene Bewertungsproblematik bei Letzteren zurückzuführen sein könnte. Allerdings zeigt sich, dass bei einer Beseitigung des Bewertungs-Lags die zeitliche Verschiebung lediglich reduziert wird, aber keinesfalls verloren geht. Die Ursache für die Lags im Renditeprozess direkter Immobilien ist also maßgeblich auf Ineffizienzen zurückzuführen, wie z.B. eine verzögerte Reaktion der Angebotsseite auf Veränderungen in der Nachfrage aufgrund langer Bauzeiten und langfristiger Mietverträge. Darüber hinaus verhindert die per se starke Heterogenität des Immobilienmarktes durch mangelnde Vergleichbarkeit, geringe Markttransparenz, lange Anlagehorizonte und hohe Transaktionskosten ein effizientes Investorenverhalten, was schließlich eine verzögerte Anpassung von Mieten und Preisen an veränderte ökonomische Rahmenbedingungen zur Folge hat (Morawski et al. 2008).

Aufgrund des Nachweises eines signifikanten Langfrist-Zusammenhangs zwischen REITs und direkten Immobilienanlagen bei gleichzeitig deutlich niedrigeren Informations- und Transaktionskosten konzentriert sich die empirische Untersuchung zum Diversifikationspotenzial von Immobilienanlagen im nächsten Abschnitt auf dieses indirekte Investmentvehikel.

\section{Der taktische und strategische Mehrwert von REITs in einem Mixed-Asset-Portfolio}

Um zu überprüfen, inwieweit REITs langfristige Diversifikationsmöglichkeiten in einem Mixed-Asset-Portfolio eröffnen, wird im Folgenden auf gemeinsame stochastische Trends in den Zeitreihen eines Mixed-Asset-Portfolios mit Hilfe der Johansen-Kointegrationanalyse getestet. Im Kontext der Portfoliotheorie bedeutet 
dies, dass die Wertentwicklung eines Vermögensportfolios bestehend aus nichtstationären Asset-Preisen mit festen Gewichten stationär ist und folglich die Assets kointegriert sind. Die Menge an Asset-Gewichten innerhalb eines solchen Portfolios leitet sich sodann aus dem Kointegrationsvektor ab. Der Johansen-Test basiert auf einer Maximum-Likelihood-Schätzung eines voll spezifizierten Vektor-FehlerKorrektur-Modells (VECM) (Johansen und Juselius 1990):

$$
\Delta X_{t}=\mu+\sum_{i=1}^{k-1} \Gamma_{i} \Delta X_{t-i}+\Pi X_{t-1}+\varepsilon_{t},
$$

mit $\Delta X_{t}$ als $(n \times 1)$-Vektor der ersten Differenzen der stochastischen Asset-Preise in Periode $t$ und $\mu$ als $(n \times 1)$-Vektor der Konstanten. $\Gamma$ als $(n \times n)$-Matrix erfasst die kurzfristige Dynamik, während die П-Matrix die Langzeitinformation enthält. Der Rang der П-Matrix muss bei Vorliegen einer oder mehrerer Kointegrationsbeziehungen zwischen der Nullmatrix und vollem Rang liegen, $0<r(\Pi)=r<n$, d.h. $r$ Linearkombinationen nicht-stationärer Variablen sind stationär bzw. es existieren $r$ Kointegrationsbeziehungen. In diesem Fall ergibt sich $\Pi=\alpha \beta^{\prime}$ als Produkt zweier Matrizen der Ordnung $n \times r$. In dieser Faktorzerlegung gibt die n-te Zeile der Feedback-Matrix $\alpha$ die Richtung und Stärke des Anpassungsprozesses an, während die $r$ Spalten von $\beta$ die Kointegrationsvektoren, d.h. die langfristigen Gleichgewichtsbeziehungen aller $n$ Variablen des vektorautoregressiven (VAR-) Modells erfassen. Um den Rang $r$ der geschätzten, langfristigen Einflussmatrix bzw. die Anzahl der Kointegrationsbeziehungen zu bestimmen, wird der Trace-Test herangezogen. Nach Cheung und Lai (1993) ist die Trace-Teststatistik im Vergleich zum Maximum-Eigenwert-Test robuster gegenüber Abweichungen von der Normalverteilung, d.h. bei Existenz von Schiefe und ExzessKurtosis.

In der Literatur wird häufig die Existenz eines gemeinsamen stochastischen Trends als mangelndes, langfristiges Diversifikationspotenzial gewertet. Erweitert man diese enge Betrachtungsweise und differenziert man zwischen taktischer und strategischer Asset Allocation nach Lucas (1997), so hängt die Bedeutung einer Kointegrationsbeziehung zwischen Asset-Preisen und REITs für die Allokationsentscheidung vom Anlagehorizont, der Umschichtungshäufigkeit des Portfolios sowie der Risikoeinstellung des Investors ab. Demzufolge werden risikoaverse Investoren im Rahmen einer strategischen Asset Allocation aus der Kenntnis der Kointegrationsbeziehungen profitieren, da kointegrierte Assets eine geringere langfristige Variabilität und somit ein geringeres Risiko aufweisen.

Die Kenntnis des Fehlerkorrekturmechanismus liefert wertvolle Informationen zur Steuerung der taktischen Asset Allocation im Portfoliomanagementprozess. Indem $X_{t}$ vorübergehend vom Gleichgewichtszustand abweicht, kann aufgrund der Funktion des Fehlerkorreturmechnismus die zukünftige Entwicklung von $X_{t}$ teilweise prognostiziert werden (Lucas 1997). Dies bedeutet, dass im Falle kointegrierter Assets Portfoliomanager die bedingten Informationen aus dem Fehlerkorrekturmechanismus ausnutzen und kurzfristige Entwicklungen antizipieren können. Wenn sich dieser Prozess jedoch über einen längeren Zeitraum erstreckt, sollten sich passive und an der langen Frist orientierte Investoren stärker am Fehlerkorrekturmechanismus als an den Kointegrationsvektoren orientieren. 


\section{Diversifikationsvorteile verbriefter Immobilienanlagen in einem Mixed-Asset-Portfolio}

Aktive Anleger können in diesem Fall hingegen die Existenz von Kointegration gänzlich vernachlässigen.

Um diesen Zusammenhang zu veranschaulichen, wird ein Investor mit einer quadratischen Risikonutzenfunktion betrachtet, d.h. bedingter Mittelwert und Varianz der Portfoliorenditen sind über den Anlagehorizont von Interesse. Des Weiteren wird angenommen, dass der Vektor der logarithmierten Asset-Preise $X_{t}$ einem VAR-Prozess der Ordnung 1 folgt:

$$
\Delta X_{t}=\mu+\Pi X_{t-1}+\varepsilon_{t} \quad \text { mit } \varepsilon_{t} \sim \text { iid }(0, \Omega)
$$

Für $\Pi=0$ resultiert das Standardmodell mit i.i.d. Renditen, während $\Pi=\alpha \beta$ ' in Gleichung (2) ein kointegriertes System von Asset-Preisen repräsentiert. ${ }^{14}$ Für einen konstanten Haltevektor $x$ in den Assets über den Anlagehorizont $H$ ergeben sich bedingter Mittelwert und Varianz wie folgt:

$$
\begin{gathered}
x^{\prime} E_{0}\left(X_{H}-X_{0}\right)=\sum_{s=0}^{H-1} x^{\prime}(I+\Pi)^{s} \mu+x^{\prime}\left[(I+\Pi)^{H}-I\right] X_{0} \\
x^{\prime} V_{0}\left(X_{H}-X_{0}\right) x=\sum_{s=0}^{H-1} x^{\prime}(I+\Pi)^{s} \Omega\left(I+\Pi^{\prime}\right)^{s} x
\end{gathered}
$$

Bei fehlender Kointegration $(\Pi=0)$ reduzieren sich die Gleichungen (3) und (4) zur Standardformulierung $H x^{\prime} \mu$ und $H x^{\prime} \Omega x$ für den Mittelwert bzw. die Varianz, anhand derer die gewohnte Mittelwert-Varianz-Analyse nach Markowitz (1952) erfolgt. Sind die Asset-Preise kointegriert $\left(\Pi=\alpha \beta^{\prime}\right)$, besitzt die MittelwertVarianz-Analyse nach wie vor ihre Gültigkeit. Allerdings resultieren nun unterschiedliche Werte für die bedingten Momente, die zu einer Verschiebung in der Mittelwert-Varianz-Grenze führen. Damit wird deutlich, dass im Rahmen der Asset Allocation die Relevanz der Kointegration vom Investmenthorizont, der Umschichtungshäufigkeit des Portfolios sowie der Risikoneigung des Investors abhängt.

Für die strategische Asset Allocation mit einem statischen langfristigen Anlagestil ohne Umschichtungen resultiert - wie oben gezeigt - eine Portfoliokonstruktion mit Gewichten entsprechend der Kointegrationsvektoren in $\beta^{\prime} X_{t-1}$. Die geringe langfristige Volatilität eines solchen Portfolios erscheint für sehr risikoaverse Investoren mit einem langen Anlagehorizont besonders attraktiv. Andererseits können Portfoliomanager im Rahmen der taktischen Asset Allocation vorübergehende Abweichungen der Asset-Preise von ihrem langfristigen Gleichgewicht antizipieren und gezielt Umschichtungen unter Vewendung der $\alpha$ Matrix vornehmen.

Das in dieser Analyse betrachtete Mixed-Asset-Portfolio setzt sich neben weltweiten REIT-Anlagen, welche durch den FTSE/NAREIT Developed Index repräsentiert werden, aus dem MSCI Weltaktienindex, dem S\&P/IFCI Composite Aktienindex für die Emerging Markets, aus dem DJ CS Hedge Funds Index, aus einem

14. Für $\Pi=0$ liegen die Eigenwerte von (I+ $\Pi)$ auf dem Einheitskreis und das Modell in Gleichung (2) reduziert sich auf einen reinen Random Walk mit Drift. 
Index für Rohstoffe (Equally Weighted Continuous Commodity Index (CCI)) sowie dem J.P. Morgan Global Government Index für globale Staatsanleihen und dem J.P. Morgan EMBI Global Composite Index für Staatsanleihen aus den Emerging Markets zusammen. Sämtliche Zeitreihen liegen als Performanceindizes (Total Return) vor und berücksichtigen somit Dividenden- und Zinserträge. Die Berechnungen zur deskriptiven Statistik in Tabelle 2 basieren auf stetigen Monatsrenditen für den Zeitraum von Januar 1994 bis Dezember 2009. Die höchste risikoadjustierte Rendite gemäß der einfachen Sharpe-Ratio erzielten Anleger im Betrachtungszeitraum durch Investments in Hedgefonds gefolgt von Anlagen in Anleihen. Aufgrund des hohen Schwankungsrisikos in den Schwellenländern weisen Aktienanlagen in diesen Märkten die schlechteste risikoadjustierte Performance aus. Mit Ausnahme von Anleihen in Industrieländern zeigen die Renditeverteilungen eine signifikante, negative Schiefe und positive Exzess-Kurtosis auf, wodurch das Risiko eines Portfolios in den Verteilungsenden deutlich höher ausfallen dürfte als dies durch die Standardabweichung zum Ausdruck kommt. Die daraus resultierende Abweichung von der Normalverteilung nach dem Jarque-Bera-Test untermauert die Verwendung der Trace-Statistik im nachfolgenden Johansen-Test auf Kointegration.

Der Kointegrationstest verlangt, dass die Zeitreihen integriert von der gleichen Ordnung sein müssen bzw. nach gleichem Grad an Differenzenbildung stationär sein müssen. In der Regel weisen Finanzmarktzeitreihen nach einmaliger Differenzenbildung einen stabilen und invertierbaren ARMA-Prozess auf, d.h. sie werden als I(1) charakterisiert. Anhang 1 zeigt die Ergebnisse des Augmented Dickey-Fuller $(1979,1981)$ (ADF)-Tests für die Zeitreihen in Levels (logarithmierte Performancekurse) und in ersten Differenzen (stetigen Renditen) der einbezogenen Assets. Mit Ausnahme der stationären Zeitreihe für Anleihen aus den Emerging Markets sind nach dem ADF-Test alle Datenreihen in den Levels nicht-stationär wie die niedrigen Werte der Teststatistik verdeutlichen. Bei Betrachtung der ersten Differenzen bzw. stetiger Renditen zeigt sich, dass die Nullhypothese einer Einheitswurzel auf dem 1\%-Signifikanzniveau abgelehnt wird. Demzufolge können mit Ausnahme der Asset-Kategorie „Anleihen aus den Emerging Markets“ die betrachteten Zeitreihen im Folgenden in die Kointegrationsanalyse einbezogen werden.

Da die Wahrscheinlichkeit eines Fehlers 2. Art bei Kointegrationstests zunimmt, wenn Strukturbrüche vorliegen, wurden sämtliche Zeitreihen nach dem QuandtAndrews-Test auf Strukturbrüche getestet. Wie die Tabelle in Anhang 2 zeigt, konnten in den Zeitreihen für den Betrachtungszeitraum keine signifikanten Strukturbrüche nachgewiesen werden. Die Bestimmung der Ordnung des unrestringierten VAR-Modells orientiert sich an den Informationskriterien Final Prediction Error (FPE), Akaike (AIC), Schwarz (SIC) und Hannan-Quinn (HQ). Sämtliche Informationskriterien deuten auf ein VAR 1. Ordnung hin, womit das VECM $k-1=0$ Differenzenterme umfasst. Des Weiteren hängt die Verteilung der Teststatistik entscheidend von der Berücksichtigung einer deterministischen Komponente in der Modellspezifikation ab. Da nicht davon auszugehen ist, dass im Mittel die Kointegrationsbeziehungen zwischen den logarithmierten Asset-Preisen null sind und langfristig von den gleichen ökonomischen Faktoren getrieben werden, wird von einem Modell mit Konstante in der Kointegrationsgleichung ausgegangen. 


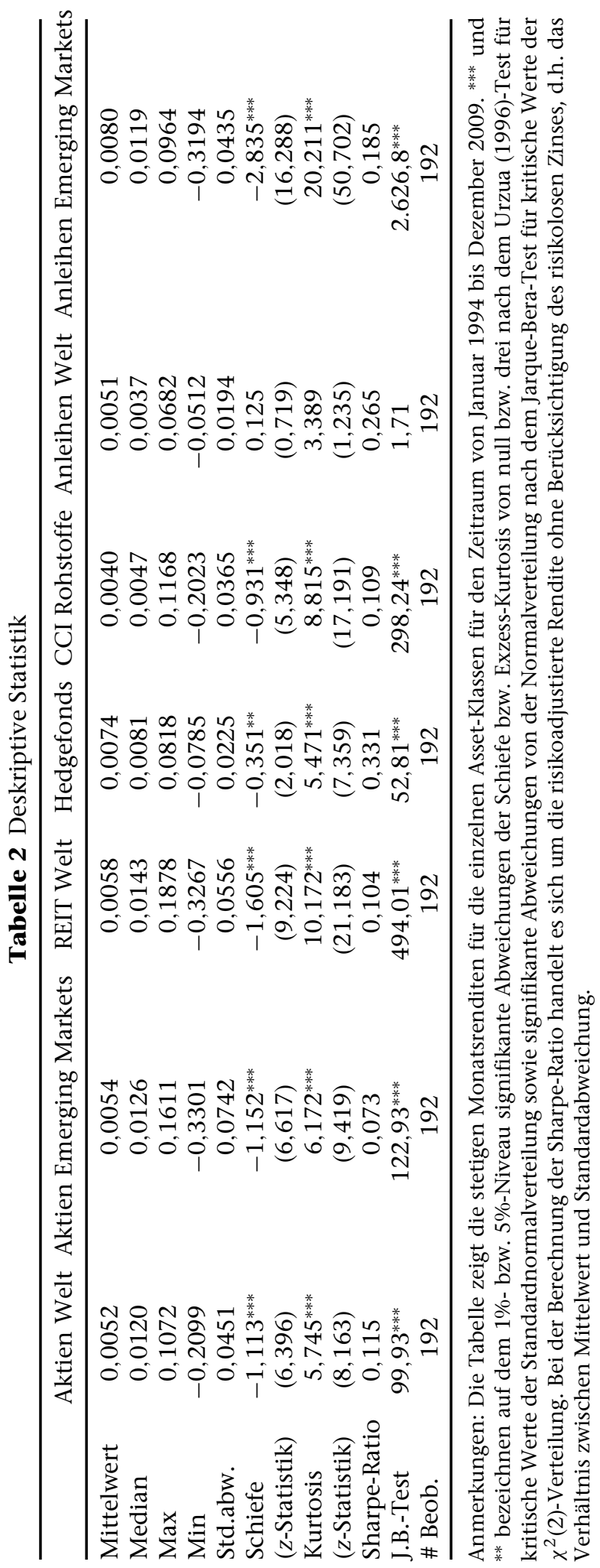


Roland Füss und Felix Schindler

Tabelle 3 Trace-Test auf Kointegration

\begin{tabular}{lrrrr}
\hline & & & \multicolumn{2}{c}{ Kritische Werte } \\
\cline { 3 - 4 } $\mathrm{H}_{0}$ & Eigenwert & Trace-Statistik & 5\%-Niveau & 1\%-Niveau \\
\hline$r=0$ & 0,2051 & $100,279^{*}$ & 94,15 & 103,18 \\
$r \leq 1$ & 0,1149 & 56,202 & 68,52 & 76,07 \\
$r \leq 2$ & 0,1065 & 32,769 & 47,21 & 54,46 \\
$r \leq 3$ & 0,0400 & 11,145 & 29,68 & 35,65 \\
$r \leq 4$ & 0,0118 & 3,300 & 15,41 & 20,04 \\
$r \leq 5$ & 0,0053 & 1,028 & 3,76 & 6,65 \\
\hline
\end{tabular}

Anmerkungen: Die Ordnung des VAR-Modells in Levels wurde auf Basis des Final Prediction Error (FPE)-, des Akaike (AIC)-, des Schwarz (SIC)- und des Hannan-Quinn (HQ)-Kriteriums auf eins festgelegt. Die Modellspezifikation erfolgt mit einer deterministischen Komponente, d.h. mit einer Konstanten in der Kointegrationsbeziehung. Die kritischen Werte für den Johansen-Test sind der Tabellierung nach Osterwald-Lenum (1992) entnommen. Die Entscheidung für die Existenz von Kointegration erfolgt nach dem Trace-Test, der nach Cheung und Lai (1993) im Vergleich zum Maximum-Eigenwert-Test robuster gegenüber Abweichungen von der Normalverteilung, d.h. bei Existenz von Schiefe und ExzessKurtosis, ist. * bezeichnet die Ablehnung der Nullhypothese fehlender Kointegration auf dem 5\%Signifikanzniveau.

Das Ergebnis der Trace-Statistik in Tabelle 3 deutet auf eine Kointegrationsbeziehung auf dem 5\%-Signifikanzniveau hin. Die Schätzung des Kointegrationsvektors normalisiert auf Aktien Welt als erste Komponente ergibt:

$$
\begin{aligned}
\text { Aktien Welt }= & 7,361+3,288 \cdot \text { Aktien EM }+0,806 \cdot \text { REIT Welt }-1,447 \cdot \text { Hedgefonds } \\
& -8,036 \cdot \text { CCI Rohstoffe }+4,908 \cdot \text { Anleihen Welt }
\end{aligned}
$$

Dabei ist der Koeffizient für die Asset-Klasse REITs in absoluten Werten am kleinsten, während Rohstoffe einen hohen negativen Koeffizienten aufweisen. Zur Überprüfung der Zusammensetzung des Kointegrationsvektors und der Anpassung der REIT-Renditen an den gemeinsamen Trend werden die Restriktionen $\beta_{\text {Aktien Welt }}=1$ und $\beta_{\text {REIT }}=0$ sowie für den Anpassungskoeffizienten $\alpha_{\text {REIT }}=0$ auferlegt. Hierbei ergibt sich eine $\chi^{2}$-Statistik von $\chi^{2}(2)=2,532$, d.h. die Nullhypothese kann nicht abgelehnt werden und REITs lassen weder einen gemeinsamen Trend noch eine signifikante Reaktion auf diesen erkennen. Demzufolge wird sich für passive, risikoaverse Investoren mit langfristigem Anlagehorizont das Portfoliorisiko durch Berücksichtigung von internationalen REIT-Märkten erhöhen, während es sich für aktive, weniger risikoaverse Investoren durch das vorhandene Diversifikationspotenzial verringern wird. Da auch der Anpassungskoeffizient der REIT-Zeitreihe nicht von null verschieden ist, resultieren für die taktische Asset Allocation zusätzliche kurzfristige Diversifikationsvorteile durch Beimischung von REITs in ein Mixed-Asset-Portfolio.

Betrachtet man abschließend die Korrelationsstrukturen zwischen REITs und den anderen Asset-Klassen für unterschiedliche Anlagehorizonte in Tabelle 4, so werden die aus der Kointegrationsanalyse gewonnenen Ergebnisse untermauert. Es wird ersichtlich, dass die Korrelationen zwischen verbrieften Immobilien und anderen Asset-Klassen bei niedriger Frequenz nicht deutlich ansteigen. Im Gegenteil, teilweise resultieren rückläufige oder nahezu konstante Korrelationen, 
Diversifikationsvorteile verbriefter Immobilienanlagen in einem Mixed-Asset-Portfolio

Tabelle 4 Korrelationskoeffizienten zwischen den Asset-Klassen und REITs

\begin{tabular}{lccrrrrr}
\hline & 1 & 3 & 6 & 9 & 12 & 18 & 24 \\
& Monat & Monate & Monate & $\begin{array}{c}\text { Monate } \\
\text { Monate }\end{array}$ & $\begin{array}{c}\text { Monate } \\
\text { Monate }\end{array}$ \\
\hline Aktien Welt & 0,762 & 0,751 & 0,724 & 0,704 & 0,722 & 0,695 & 0,600 \\
Hedgefonds & 0,722 & 0,699 & 0,735 & 0,801 & 0,741 & 0,807 & 0,730 \\
CCI Rohstoffe & 0,369 & 0,580 & 0,626 & 0,668 & 0,734 & 0,670 & 0,564 \\
Anleihen Welt & 0,231 & 0,401 & 0,581 & 0,669 & 0,648 & 0,719 & 0,658 \\
\hline
\end{tabular}

was auf langfristig stabile und günstige Diversifikationseffekte durch Investments in internationale REITs schließen lässt.

\section{Zusammenfassung und Ausblick}

Der vorliegende Beitrag beschäftigt sich mit dem Diversifikationspotenzial verbriefter Immobilienanlagen in einem Mixed-Asset-Portfolio. Anhand der Diskussion unterschiedlicher Immobilienanlageformen nach ihrer Zuordnung zu Eigen- und Fremdkapital sowie hinsichtlich ihrer Einordnung in „börsengehandelte” bzw. „nicht börsengehandelte” Immobilieninvestments zeigt sich unter Berücksichtigung der Rendite-Risiko-Eigenschaften die Vorteilhaftigkeit der Vermögensanlageklasse Real Estate Investments Trusts (REITs). Die durch eine Börsennotierung verbundene höhere Korrelation von REITs zum Aktienmarkt während der 1990er Jahre hat in der letzten Dekade deutlich abgenommen, womit REITs zunehmend als eigenständige Asset-Klasse betrachtet werden können. Darüber hinaus bleibt das Exposure zum direkten Immobilienmarkt - zumindest in den langen Frist - erhalten, wie zahlreiche neuere empirische Untersuchungen zeigen können. Dabei ist zu erkennen, dass die Entwicklung von REITs derjenigen von direkten Immobilienanlagen voraus läuft, was u.a. auf Marktineffizienzen und auf die Bewertungsproblematik am direkten Immobilienmarkt zurückzuführen ist.

Des Weiteren kann unter Verwendung des Johansen-Kointegrationstests eine Erhöhung des Diversifikationspotenzials durch Einbeziehung internationaler REITs in die strategische Asset Allocation eines Mixed-Asset-Portfolios nachgewiesen werden, indem REITs in der Betrachtungsperiode keinen gemeinsamen langfristigen Trend mit den anderen Anlagekategorien aufweisen. Folglich bietet diese Anlageklasse gerade aktiven Anlegern sowie Investoren mit langfristigem Anlagehorizont ein adäquates Diversifikationspotenzial bei Vereinnahmung kontinuierlicher Zahlungsströme.

\section{Literaturverzeichnis}

Alda, W. und J. Lassen (1998), Kapitalanlagegesellschaften, in: K.-W. Schulte, S. Bone-Winkel und M. Thomas (Hrsg.), Handbuch Immobilien-Investition. Müller, Köln 1998, 2, vollständig überarbeitete Auflage 2005, 87-121.

Brueggeman, W. und J.D. Fisher (2008), Real Estate Finance and Investments. 13th ed., Irwin McGraw-Hill, Boston.

BVI (2010), Investment 2010, Daten, Fakten, Entwicklungen. Frankfurt am Main.

Case, K.E. und R.J. Shiller (1989), The Efficiency of the Market for Single-Family Homes, American Economic Review 79, 125-137. 


\section{Roland Füss und Felix Schindler}

Chan, S.H., J. Erickson und K. Wang (2003), Real Estate Investment Trusts - Structure, Performance, and Investment Opportunities. Oxford University Press, Oxford.

Cheung, Y.-W. und K.S. Lai (1993), Finite-Sample Sizes of Johansen's Likelihood Ratio Tests for Cointegration, Oxford Bulletin of Economic and Statistics 55, 313-328.

Demary, M., P. Gans, R. Meng, A. Schmitz-Veltin, M. Voigtländer und P. Westerheide (2009), Wirtschaftsfaktor Immobilie - Die Immobilienmärkte aus gesamtwirtschaftlicher Perspektive, Zeitschrift für Immobilienökonomie, Sonderausgabe 2009.

Dickey, D.A. und W.A. Fuller (1979), Distribution of the Estimators for Autoregressive Time Series with a Unit Root, Journal of the American Statistical Association 74, 427-431.

Dickey, D.A. und W.A. Fuller (1981), Likelihood Ratio Tests for Autoregressive Time Series with a Unit Root, Econometrica 49, 1057-1072.

Downs, D.H. und D.J. Hartzell (1995), Real Estate Investment Trusts, in: J.L. Pagliari (Hrsg.), The Handbook of Real Estate Portfolio Management. Irwin McGraw-Hill, Boston, 597634.

Eichholtz, P.M.A. und D.J. Hartzell (1996), Property Shares, Appraisals and the Stock Market: An International Perspective, Journal of Real Estate Finance and Economics 12, 163-178.

Eichholtz, P.M.A. und N. Kok (2007), The EU REIT and the Internal Market for Real Estate, Research Report, Brüssel.

Ellwanger und Geiger (2000), Europäische Immobilien-Aktien. Stuttgart.

EPRA (2009), Global REIT Survey - A Comparison of the Major REIT Regimes in the World. Brüssel.

Fass, P.M., M.E. Shaff und D.B. Zief (2005), Real Estate Investment Trusts Handbook - A Pass Through Entity to Own and Operate Real Estate and Make Mortgage Loans. Thomson, St. Paul.

Fisher, J.D. und D.M. Geltner (2000), De-Lagging the NCREIF Index: Transaction Prices and Reverse-Engineering, Real Estate Finance 17, 7-22.

Gau, G. (1984), Weak Form Tests of the Efficiency of Real Estate Investment Markets, The Financial Review 19, 301-320.

Geltner, D.M. (1991), Smoothing in Appraisal-Based Returns, Journal of Real Estate Finance and Economics 4, 327-345.

Geltner, D.M. (1993), Temporal Aggregation in Real Estate Return Indices, Journal of American Real Estate and Urban Economics Association (AREUEA) 4, 327-345.

Geltner, D.M., J.V. Rodriguez und D. O'Connor (1995), The Similar Genetics of Public and Private Real Estate and the Optimal Long-Horizon Portfolio Mix, Real Estate Finance 13, $13-25$.

Genesove, D. und C.J. Mayer (1997), Equity and Time to Sale in the Real Estate Markets, American Economic Review 87, 255-276.

Gordon, J.N. (1994), The Real Estate Capital Markets Matrix: A Paradigm Approach, Real Estate Finance 11, 7-15.

Hansen, B.E. (1997), Approximate Asymptotic P Values for Structural-Change Tests, Journal of Business and Economic Statistics 15, 60-67.

Hudson-Wilson, S., J.N. Gordon, F.J. Fabozzi, M.J.P. Anson und S.M. Giliberto (2005), Why Real Estate? - And How? Where And When?, Journal of Portfolio Management 32, 12-22.

Johansen, S. (1988), Statistical Analysis of Cointegration Vectors, Journal of Economic Dynamics and Control 12, 231-206.

Johansen, S. (1991), Estimation and Hypothesis Testing of Cointegration Vectors in Gaussian Vector Autoregressive Models, Econometrica 59, 1551-1580.

Johansen, S. und K. Juselius (1990), Maximum Likelihood Estimation and Inference on Cointegration: With Applications to the Demand for Money, Oxford Bulletin of Economic and Statistics 52, 169-210.

Lee, M.-L. und K.C.H. Chiang (2004), Substitutability between Equity REITs and Mortgage REITs, Journal of Real Estate Research 26, 95-113.

Lucas, A. (1997), Strategic and Tactical Asset Allocation and the Effect of Long-run Equilibrium Relations, Research Memorandum, Vrije University Amsterdam.

Markowitz, H.M. (1952), Portfolio Selection, Journal of Finance 7, 77-91. 


\section{Diversifikationsvorteile verbriefter Immobilienanlagen in einem Mixed-Asset-Portfolio}

Morawski, J., H. Rehkugler und R. Füss (2008), The Nature of Listed Real Estate Companies: Property or Equity Market?, Financial Markets and Portfolio Management 22, 101-126.

Mueller, C. (2010), Commercial Real Estate as Inflation Hedge, Dividend Capital Research and Dividend Capital Securities. Denver.

Oikarinen, E., M. Hoesli und C. Serrano (2009), Linkage between Direct and Securitized Real Estate, Swiss Finance Institute Research Paper Series No. 09-26, Zürich.

Osterwald-Lenum, M. (1992), A Note with Quantiles of the Asymptotic Distribution of the Maximum Likelihood Cointegration Rank Test Statistics, Oxford Bulletin of Economic and Statistics 54, 461-472.

Pagliari, J.L., K.A. Scherer und R.T. Monopoli (2003), Public versus Private Real Estate Equities, Journal of Portfolio Management 30, 101-111.

Pagliari, J.L., K.A. Scherer und R.T. Monopoli (2005), Public versus Private Real Estate Equities: A More Refined, Long-Term Comparison, Real Estate Economics 33, 147-187.

Paramor, G. und K. Wright (2006), The Four Quadrant Investment Model, 3rd Quarter, Sydney.

Phyrr, S.A., S.E. Roulac und W.L. Born (1999), Real Estate Cycles and Their Strategic Implications for Investors and Portfolio Managers in the Global Economy, Journal of Real Estate Research 18, 7-68.

Quan, D.C. und J.M. Quigley (1989), Inferring an Investment Return Series for Real Estate from Observations on Sales, Journal of American Real Estate and Urban Economics Association (AREUEA) 17, 218-230.

Rehkugler, H. (2000), Die Immobilien-AG als attraktive Kapitalanlage - Chancen für Unternehmen und Investoren, Finanzbetrieb, 2. Jg., Nr. 4, 230-239.

Riddiough, T.J., M. Moriartry und P.J. Yeatman (2005), Privately Versus Publicly Held Asset Investment Performance, Real Estate Economics 33, 121-146.

Schindler, F. (2011), The Predictability and Persistence of the Price Movements of the S\&P/Case-Shiller House Price Indices, Journal of Real Estate Finance and Economics, forthcoming.

Schmidt-von Rhein, A. (1996), Die Moderne Portfoliotheorie im praktischen Wertpapiermanagement - Eine theoretische und empirische Analyse aus Sicht privater Kapitalanleger. Uhlenbruch, Bad Soden/Ts.

Schwert, G.W. (1989), Tests for Unit Roots: A Monte Carlo Investigation, Journal of Business and Economic Statistics 7, 147-159.

Stein, J. (1995), Prices and Trading Volume in the Housing Market: A Model with Downpayment Effects, Quarterly Journal of Economics 11, 379-406.

Tirtiroglu, D. (1992), Efficiency in Housing Markets: Temporal and Spatial Dimensions, Journal of Housing Economics 2, 276-292.

Urzua, C.M. (1996), On the Correct Use of Omnibus Tests for Normality, Economics Letters 53, 247-251.

Wurtzebach, C.H., G.R. Mueller und D. Machi (1991), The Impact of Inflation and Vacancy on Real Estate Returns, Journal of Real Estate Research 6, 153-168.

Abstract: This article examines whether international investors benefit from adding real estate investment trusts (REITs) to a mixed asset portfolio consisting of global stocks, bonds, hedge funds, and commodities. Previous literature has shown that REITs provide a strong co-movement with direct real estate in the long run. We therefore test the diversification potential of international REITs within the strategic asset allocation. Using the Johansen cointegration technique, we show that there is no long-term co-movement between REITs and the other asset classes in the period from January 1990 to December 2009. Thus, the empirical evidence suggests that REITs improve the diversification potential for active investors and those with a long-term investment horizon by simultaneously generating continuous cash flows. 
Roland Füss und Felix Schindler

Anhang 1: Augmented Dickey-Fuller (ADF) Einheitswurzeltest

\begin{tabular}{|c|c|c|c|}
\hline \multirow[b]{2}{*}{ Indizes } & $\mathrm{ADF}$ & $\mathrm{ADF}_{\mathrm{C}}$ & $\mathrm{ADF}_{\mathrm{CT}}$ \\
\hline & \multicolumn{3}{|c|}{ in Levels } \\
\hline Aktien Welt & $1,148(1)$ & & \\
\hline (AIC) $[\mathrm{SIC}]$ & $(-3,384)[-3,350]$ & & \\
\hline Aktien Emerging Markets & $0,9512(6)$ & & \\
\hline (AIC) $[\mathrm{SIC}]$ & $(-2,355)[-2,234]$ & & \\
\hline REIT Welt & & & $-2,547(4)$ \\
\hline (AIC) $[\mathrm{SIC}]$ & & & $(-3,044)[-2,924]$ \\
\hline Hedgefonds & $2,315(8)$ & & \\
\hline (AIC) $[\mathrm{SIC}]$ & $(-4,751)[-4,594]$ & & \\
\hline CCI - Rohstoffe & $1,337(12)$ & & \\
\hline (AIC) $[\mathrm{SIC}]$ & $(-3,766)[-3,535]$ & & \\
\hline Anleihen Welt & $3,059(12)$ & & \\
\hline (AIC) $[\mathrm{SIC}]$ & $(-5,034)[-4,803]$ & & \\
\hline \multirow[t]{2}{*}{$\begin{array}{l}\text { Anleihen Emerging Markets } \\
\text { (AIC) }[\text { SIC] }\end{array}$} & & & $\begin{array}{c}-\mathbf{4 , 1 1 7}(\mathbf{1 2})^{* * *} \\
(-3,513)[-3,247]\end{array}$ \\
\hline & \multicolumn{3}{|c|}{ in ersten Differenzen } \\
\hline $\begin{array}{l}\text { Aktien Welt } \\
(\mathrm{AIC})[\mathrm{SIC}]\end{array}$ & & $\begin{array}{c}\mathbf{- 1 1 , 4 2 4}(\mathbf{0})^{* * * *} \\
(-3,385)[-3,351]\end{array}$ & \\
\hline $\begin{array}{l}\text { Aktien Emerging Markets } \\
\text { (AIC) }[\mathrm{SIC}]\end{array}$ & $\begin{array}{c}-\mathbf{4 , 7 6 6}(\mathbf{6})^{* * *} \\
(-2,366)[-2,244]\end{array}$ & & \\
\hline REIT Welt & $-5,266(5)^{* * *}$ & & \\
\hline (AIC) $[\mathrm{SIC}]$ & $(-3,027)[-2,923]$ & & \\
\hline $\begin{array}{l}\text { Hedgefonds } \\
\text { (AIC) }[\mathrm{SIC}]\end{array}$ & & & $\begin{array}{c}-\mathbf{1 1}, \mathbf{0 2 3}(\mathbf{0})^{* * *} \\
(-4,779)[-4,728\end{array}$ \\
\hline CCI - Rohstoffe & & $-4,502(11)^{* * *}$ & \\
\hline (AIC) $[\mathrm{SIC}]$ & & $(-3,766)[-3,535]$ & \\
\hline Anleihen Welt & & $-4,245(11)^{* * *}$ & \\
\hline (AIC) $[\mathrm{SIC}]$ & & $(-5,035)[-4,805]$ & \\
\hline Anleihen Emerging Markets & & $-3,941(12)^{* * *}$ & \\
\hline (AIC) $[\mathrm{SIC}]$ & & $(-3,450)[-3,201]$ & \\
\hline
\end{tabular}

Anmerkungen: Die Tabelle zeigt die Teststatistik nach dem Augmented Dickey-Fuller (1979, 1981) (ADF)-

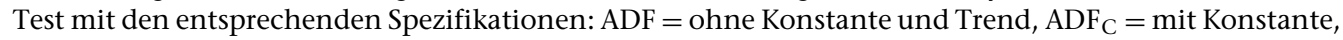
$\mathrm{ADF}_{\mathrm{CT}}=$ mit Konstante und Trend. Die Lag-Länge in Klammern (fettgedruckt) wurde nach dem Akaike Informationskriterium (AIC) und dem Schwarz Informationskriterium [SIC] bestimmt, wobei letzteres als Entscheidungskriterium für die Modellspezifikation verwendet wird. Der maximale Startwert zur Bestimmung der Lag-Länge wurde nach Schwert (1989) mit $T=193$ Beobachtungen auf $p_{\max }=12$. $(T / 100)^{1 / 4} \approx 14$ festgelegt. Dabei wurde nach dem unverzerrten General-to-Specific-Ansatz vorgegangen, d.h. die Teststatistik für die verschiedenen Spezifikationen mit einer Lag-Struktur gewählt, bei der der höchste Lag-Term auf dem 10\%-Niveau signifikant ist. ***, ** und * für Signifikanz auf dem 1\%-, 5\%und 10\%-Niveau (mit Einheitswurzel als Nullhypothese). 


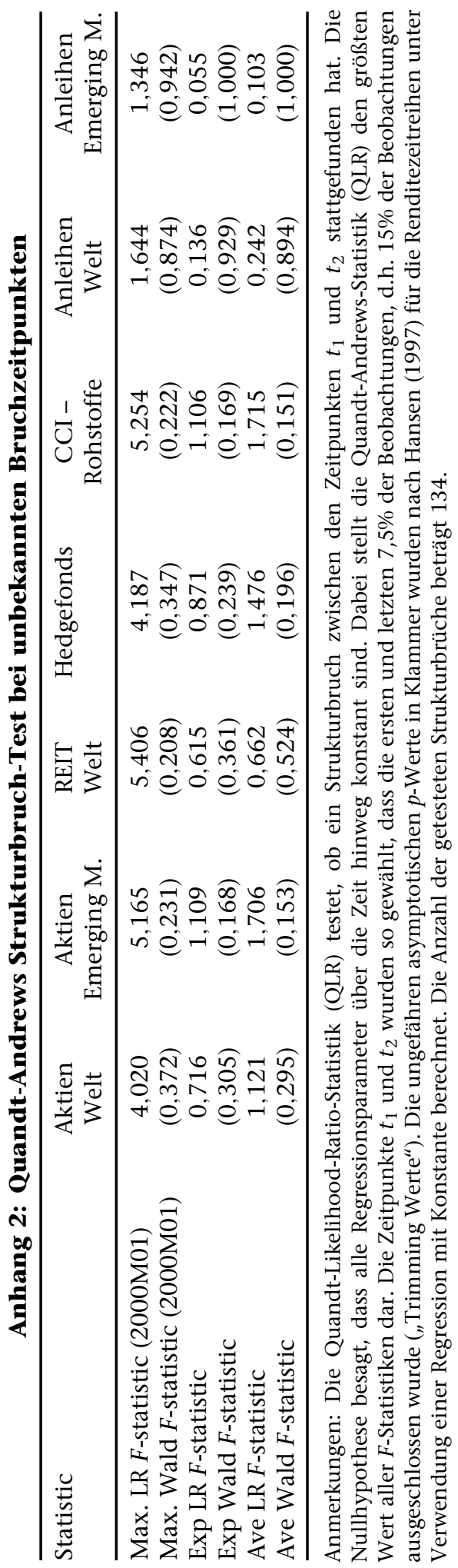

